Nervenarzt 2022 · 93:503-505

https://doi.org/10.1007/s00115-021-01239-1

Angenommen: 26. Oktober 2021

Online publiziert: 3. Dezember 2021

(c) Der/die Autor(en) 2021

\section{Behandlung dissoziativer Symptome mit Nalmefen bei Patienten mit Borderline- Persönlichkeitsstörung und komplexer posttraumatischer Belastungsstörung}

\author{
Frank Enning · Christian Schmahl
}

Klinik für Psychosomatik und Psychotherapeutische Medizin, Zentralinstitut für Seelische Gesundheit, Medizinische Fakultät Mannheim, Universität Heidelberg, Mannheim, Deutschland

\section{Hintergrund}

Dissoziative Phänomene einschließlich Flashbacks und dissoziative Anfälle sind häufige und therapiehemmende Probleme bei Patienten mit BorderlinePersönlichkeitsstörung und komplexer Posttraumatischer Belastungsstörung (PTBS). Es wird angenommen, dass das Endogene Opioidsystem (EOS) dabei eine wichtige Rolle spielt. Bezüglich des EOS wurde bisher nur Naltrexon und sein Wirkmechanismus bei der Behandlung dissoziativer Symptome untersucht. Nalmefen ist ein weiterer Opioid-Antagonist und wir untersuchten seine Wirkung im Rahmen eines individuellen Behandlungsversuchs bei Patienten mit Borderline-Persönlichkeitsstörung, komplexer PTBS und schweren dissoziativen Symptomen. Nalmefen zeigte vielversprechende Ergebnisse als mögliche Alternative zu Naltrexon, insbesondere bei Unverträglichkeit oder unzureichender Wirksamkeit.

Ein klinisch relevantes Symptomcluster der Borderline-Persönlichkeitsstörung (BPS) und der komplexen PTBS ist die Dissoziation, die im DSM-Item „Vorübergehende, stressbedingte paranoide Vorstellungen oder schwere dissoziative Symptome" enthalten ist. Zu den dissoziativen Phänomenen gehören Derealisation, Depersonalisation, Analgesie und Veränderungen der
Wahrnehmung [1]. Nichtsuizidale Selbstverletzungen werden häufig eingesetzt, um diese Phänomene zu beenden. Das EOS ist an der Vermittlung von Stressreaktionen und an endogenen analgetischen Mechanismen beteiligt [2]. Die Exposition gegenüber Stress kann zur Freisetzung von Opioiden und zur Entwicklung einer stressinduzierten Analgesie führen [3]. Naltrexon kann diese Analgesie bei Patienten mit posttraumatischer Belastungsstörung (PTBS) blockieren [4]. Es gibt mehrere Fallberichte über den erfolgreichen Einsatz von Naltrexon zur Behandlung von Selbstverletzungen und Flashbacks [5-7]. In einer offenen Studie wurde ein signifikanter Rückgang der dissoziativen Symptome bei Patienten mit BPD unter Behandlung mit Naltrexon beobachtet [8]. Eine kleine randomisiertkontrollierte Studie konnte jedoch keine signifikante Wirkung von Naltrexon bei dissoziativen Symptomen bestätigen [9]. Nalmefen zeigt sowohl gemischte agonistische als auch antagonistische Effekte am к-Opioid-Rezeptor. Die antagonistische Wirkung besteht in seiner Fähigkeit, die dysphorisierende Wirkung des K-Dynorphin-Liganden zu blockieren [10]. Eine Wirkung höherer Dosen von Nalmefen zur Behandlung dissoziativer Symptome bei der PTBS wurde bereits früher berichtet [11]. 


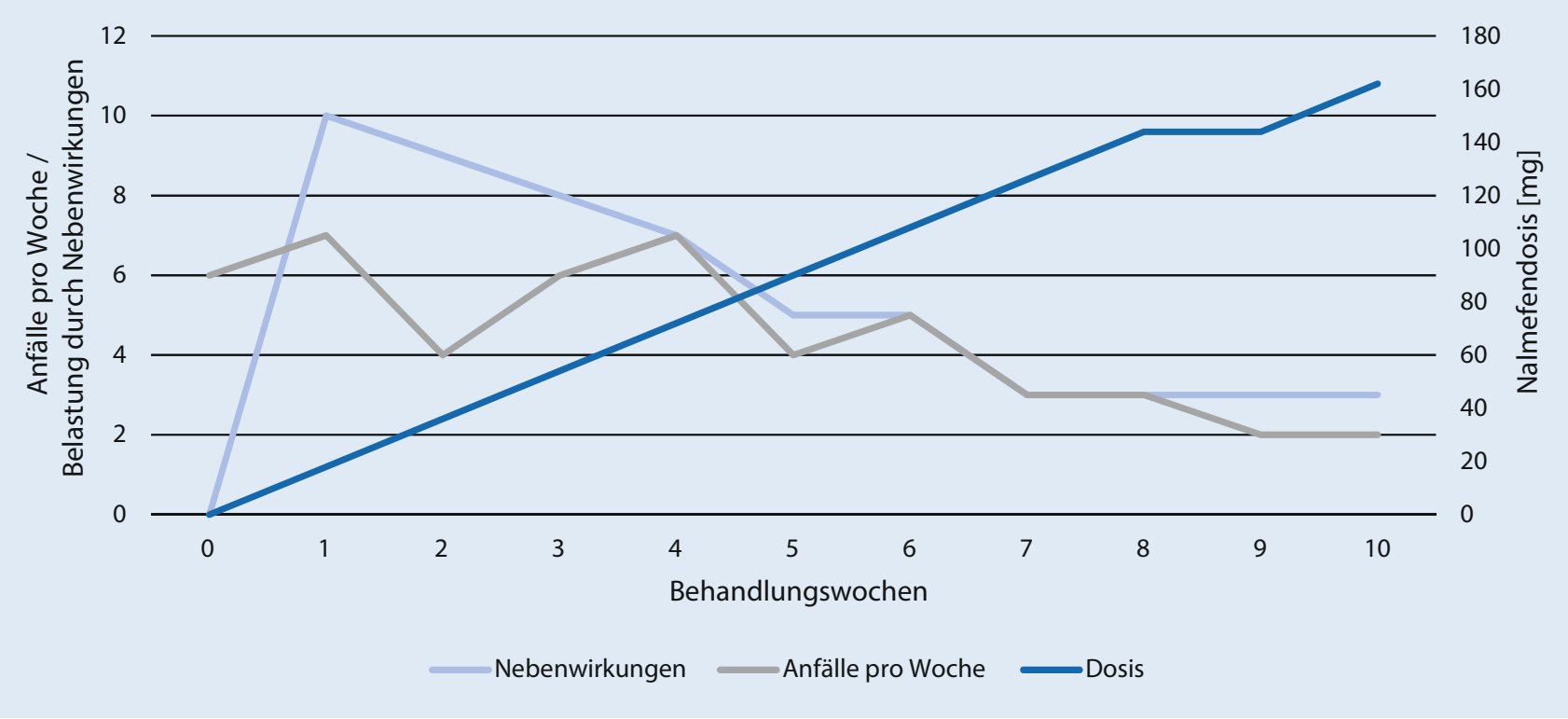

Abb. 1 ॥ Selbst eingeschätzte Symptomschwere und Intensität der Nebenwirkungen

\section{Falldarstellung}

Eine 22-jährige Studentin mit diagnostizierter BPS (ICD-10: F60.31) und PTBS (ICD: F43.1), nach körperlicher und sexueller Gewalt durch den Vater vom 4. bis zum 18. Lebensjahr, litt neben starken Stimmungsschwankungen, Leeregefühlen, Suizidideationen, Selbstverletzungen durch Verbrühen, Intrusionen und Alpträumen an massiven dissoziativen Symptomen. Neben einer Medikation mit L-Thyroxin $75 \mu \mathrm{g} / \mathrm{Tag}$ bei latenter Hypothyreose wurde der Patientin aufgrund täglicher dissoziativer Anfälle Valproinsäure in einer Dosierung von $1000 \mathrm{mg} / \mathrm{Tag}$ verabreicht, was keine signifikanten Effekte zeigte. Zu Beginn der ambulanten psychiatrischen Behandlung berichtete die Patientin über tägliche dissoziative Anfälle. Diese Symptome machten es ihr unmöglich, eine Tagesstruktur aufzubauen oder ihre Berufsausbildung zu absolvieren. Auch andere Medikationsversuche mit Sertralin und Tiaprid waren erfolglos gewesen.

Nalmefen wurde mit $18 \mathrm{mg} / \mathrm{Tag}$ begonnen und im Laufe der Behandlung auf insgesamt $162 \mathrm{mg} /$ Tag gesteigert. Wie bereits früher beschrieben [11], zeigte die Patientin bei niedriger Dosierung erhebliche $\mathrm{Ne}$ benwirkungen (z. B. Kopfschmerzen, Übelkeit und Schwindel), die mit Erhöhung der Dosis nachließen und bei $162 \mathrm{mg} / \mathrm{Tag}$ fast vollständig aufhörten. Gleichzeitig kam es zu einer deutlichen Reduktion der dissoziativen Anfälle, sowohl in der Häufigkeit als auch in der Dauer und Intensität (• Abb. 1).

Neben dieser Patientin verabreichten wir Nalmefen bei 16 stationären Patientinnen mit BPS und komplexer PTBS und ausgeprägter dissoziativer Symptomatik. Bei 4 Patientinnen musste die Behandlung aufgrund der oben beschriebenen Nebenwirkungen bei einer Dosis von $18 \mathrm{mg} / \mathrm{Tag}$, bei einer weiteren Patientin bei einer Dosis von $36 \mathrm{mg} / \mathrm{Tag}$ abgebrochen werden. Bei 4 Patientinnen konnte die Behandlung mit $36 \mathrm{mg} / \mathrm{Tag}$ ohne nennenswerte Nebenwirkungen durchgeführt werden. In 5 Fällen wurde die Behandlung mit $54 \mathrm{mg}$ ohne nennenswerte Nebenwirkungen durchgeführt. In 2 Fällen traten bei $18 \mathrm{mg} / \mathrm{Tag}$ deutliche Nebenwirkungen in Form von Übelkeit und Schwindel auf, waren aber nach Erhöhung der Dosis auf $54 \mathrm{mg} / \mathrm{Tag}$ vollständig rückläufig. Von den insgesamt 17 mit Nalmefen behandelten Patientinnen zeigte sich bei 13 Patienten im Laufe der Therapie eine relevante Reduktion dissoziativer Phänomene (• Tab. 1).

\section{Diskussion und Schluss- folgerungen}

Die Wirkung des Opioidrezeptorantagonisten Nalmefen bei Patientinnen mit BPS und ausgeprägten dissoziativen Symptomen unterstützt die Hypothese einer Be- teiligung des endogenen Opioidsystems an diesen Phänomenen [12]. Unsere Ergebnisse stehen im Einklang mit denen, die bereits früher bei Veteranen mit PTBS berichtet wurden [11]. Im Gegensatz zu Naltrexon scheint Nalmefen eine paradoxe Dosis-Nebenwirkungs-Beziehung in relevantem Ausmaß zu verursachen. Dies ist eine Implikation, die bei der klinischen Anwendung dieser Substanz beachtet werden sollte. Da dem Symptomkomplex Dissoziation eine zentrale Rolle als Prädiktor für den Erfolg der Psychotherapie der BPS und der komplexen PTBS zukommt [13, 14], stellen Opioidrezeptorantagonisten in einem multimodalen therapeutischen Ansatz eine wichtige medikamentöse Therapieoption dar [15]. Hier kann Nalmefen als Alternative zu Naltrexon gesehen werden, insbesondere bei Unverträglichkeit oder unzureichender Wirksamkeit.

\section{Korrespondenzadresse}

\section{Dr. Frank Enning}

Klinik für Psychosomatik und Psychotherapeutische Medizin, Zentralinstitut für Seelische Gesundheit, Medizinische Fakultät Mannheim, Universität Heidelberg J5, 68159 Mannheim, Deutschland frank.enning@zi-mannheim.de 


\begin{tabular}{|c|c|c|}
\hline Dosis & $\begin{array}{l}\text { Wirkung auf dissoziative } \\
\text { Symptomatik }\end{array}$ & Nebenwirkungen \\
\hline $18 \mathrm{mg} / \mathrm{Tag}$ & Dtl. Besserung & Keine \\
\hline $54 \mathrm{mg} / \mathrm{Tag}$ & Dtl. Besserung & Unter $18 \mathrm{mg}$ UAW, unter $54 \mathrm{mg} /$ Tag keine UAW \\
\hline $54 \mathrm{mg} / \mathrm{Tag}$ & Dtl. Besserung & Unter $18 \mathrm{mg}$ UAW, unter $54 \mathrm{mg} /$ Tag keine UAW \\
\hline $36 \mathrm{mg} / \mathrm{Tag}$ & Dtl. Besserung & Keine \\
\hline $54 \mathrm{mg} / \mathrm{Tag}$ & Dtl. Besserung & Keine \\
\hline $54 \mathrm{mg} / \mathrm{Tag}$ & Kaum Besserung & Keine \\
\hline $36 \mathrm{mg} / \mathrm{Tag}$ & Dtl. Besserung & Keine \\
\hline $72 \mathrm{mg} / \mathrm{Tag}$ & Dtl. Besserung & Keine \\
\hline $36 \mathrm{mg} / \mathrm{Tag}$ & Dtl. Besserung & Keine \\
\hline $18 \mathrm{mg} / \mathrm{Tag}$ & Dtl. Besserung & Keine \\
\hline $36 \mathrm{mg} / \mathrm{Tag}$ & Dtl. Besserung & Keine \\
\hline $54 \mathrm{mg} / \mathrm{Tag}$ & Dtl. Besserung & Keine \\
\hline $36 \mathrm{mg} / \mathrm{Tag}$ & Abgesetzt & Übelkeit u. Schwindel \\
\hline $18 \mathrm{mg} / \mathrm{Tag}$ & Abgesetzt & Übelkeit u. Schwindel \\
\hline $18 \mathrm{mg} / \mathrm{Tag}$ & Abgesetzt & Übelkeit u. Schwindel \\
\hline $18 \mathrm{mg} / \mathrm{Tag}$ & Abgesetzt & Übelkeit u. Schwindel \\
\hline
\end{tabular}

13. Kleindienst N, Limberger MF, Ebner-Priemer UW, Keibel-Mauchnik J, Dyer A, Berger M, Schmahl C, Bohus M (2011) Dissociation predicts poor response to dialectial behavioral therapy in female patients with borderline personality disorder. JPers Disord 25(4):432-447

14. Kleindienst N, Priebe K, Görg N, Dyer A, Steil R, Lyssenko L, Winter D, Schmahl C, Bohus M (2016) State dissociation moderates response to dialectical behavior therapy for posttraumatic stress disorder in women with and without borderline personality disorder. Eur J Psychotraumatol 7:30375

15. Timaus C, Meiser M, Wiltfang J, Bandelow B, Wedekind D (2021) Efficacy of naltrexone in borderline personality disorder, a retrospective analysis in inpatients. Hum Psychopharmacol. https://doi.org/10.1002/hup.2800

Danksagung. Die Autoren danken Hillel Glover, $M D$, für die Beratung und das kritische Lesen des Manuskripts.

Funding. Open Access funding enabled and organized by Projekt DEAL.

Interessenkonflikt. F. Enning und C. Schmahl geben an, dass kein Interessenkonflikt besteht.

Open Access. Dieser Artikel wird unter der Creative Commons Namensnennung 4.0 International Lizenz veröffentlicht, welche die Nutzung, Vervielfältigung, Bearbeitung, Verbreitung und Wiedergabe in jeglichem Medium und Format erlaubt, sofern Sie den/die ursprünglichen Autor(en) und die Quelle ordnungsgemäß nennen, einen Link zur Creative Commons Lizenz beifügen und angeben, ob Änderungen vorgenommen wurden.

Die in diesem Artikel enthaltenen Bilder und sonstiges Drittmaterial unterliegen ebenfalls der genannten Creative Commons Lizenz, sofern sich aus der Abbildungslegende nichts anderes ergibt. Sofern das betreffende Material nicht unter der genannten Creative Commons Lizenz steht und die betreffende Handlung nicht nach gesetzlichen Vorschriften erlaubt ist, ist für die oben aufgeführten Weiterverwendungen des Materials die Einwilligung des jeweiligen Rechteinhabers einzuholen.

Weitere Details zur Lizenz entnehmen Sie bitte der Lizenzinformation auf http://creativecommons.org/ licenses/by/4.0/deed.de.

\section{Literatur}

1. Krystal JH, Bennett AL, Bremner JD, Southwick SM, Charney DS (1995) Toward a cognitive neuroscience of dissociation and altered memory functions in posttraumatic stress disorder. In: Friedman MJ, Charney DS, Deutsch AY (Hrsg) Neurobiological and clinical consequences of stress. LippincottRaven, Philadelphia, S238-269

2. Amir S, Brown ZW, Amit A (1980) The role of endorphins in stress: evidence and speculations. Neurosci Biobehav Rev 4(1):77-86

3. Terman GW, Shavit Y, Lewis JW, Cannon JT, Liebeskind JC (1984) Intrinsic mechanisms of pain inhibition: activation by stress. Science 226(4680):1270-1277

4. Roth AS, Ostroff RB, Hoffman RE (1996) Naltrexone as a treatment for repetitive self-injurious behavior: an open-label trial. JClin Psychiatry 57(6):233-237

5. Winchel RM, Stanley M (1991) Self-injurious behavior: a review of the behavior and biology of self-mutilation. Am J Psychiatry 148(3):306-317

6. Pitman RK, van der Kolk BA, Orr SP, Greenberg MS (1990) Naloxone-reversible analgesic reponse to combat-related stimuli in post traumatic stress disorder. Arch Gen Psychiatry 47(6):541-544

7. Bills LJ, Kreisler K (1993) Treatment of flashbacks with naltrexone. Am J Psychiatry 150(9):1430

8. Schmahl C, Stiglmayr C, Böhme R, Bohus M (1999) Behandlung von dissoziativen Symptomen bei Borderline-Persönlichkeitsstörungen mit Naltrexon. Nervenarzt 70:262-264

9. Schmahl C, Kleindienst N, Limberger M, Ludäscher P, Mauchnik J, Deibler P, Brünen S, Hiemke C, LiebK, HerpertzS, Reicherzer M, Berger M, Bohus M (2012) Evaluation of naltrexone for dissociative symptoms in borderline personality disorder. Int Clin Psychopharmacol 27:61-68

10. Bart G, Schluger JH, Borg L, Ho A, Bidlack JM, Kreek MJ (2005) Nalmefene induced elevation in serum prolactin in normal human volunteers: partial kappa opioid agonist activity? Neuropsychopharmacology 30(12):2254-2262

11. Glover H (1993) A preliminary trial of Nalmefene for the treatment of emotional numbing in combat veterans with post-traumatic stress disorder. Isr J Psychiatry Relat Sci 30(4):255-263

12. BandelowB, SchmahlC, Falkai P, WedekindD(2010) Borderline personality disorder: a dysregulation of the endogenous opioid system? Psychol Rev 117:623-636 